\title{
Controllable broadband multicolour single-mode polarized laser in a dye-assembled homoepitaxial MOF microcrystal
}

\author{
Huajun He(i) ${ }^{1,3}$, Yuanjing Cui ${ }^{1}$, Hongjun Li ${ }^{1}$, Kai Shao ${ }^{1}$, Banglin Chen ${ }^{2}$ and Guodong Qian ${ }^{1}$
}

\begin{abstract}
Multicolour single-mode polarized microlasers with visible to near-infrared output have very important applications in photonic integration and multimodal biochemical sensing/imaging but are very difficult to realize. Here, we demonstrate a single crystal with multiple segments based on the host-guest metal-organic framework ZJU-68 hierarchically hybridized with different dye molecules generating controllable single-mode green, red, and nearinfrared lasing, with the lasing mode mechanism revealed by computational simulation. The segmented and oriented assembly of different dye molecules within the ZJU-68 microcrystal causes it to act as a shortened resonator, enabling us to achieve dynamically controllable multicolour single-mode lasing with a low three-colour-lasing threshold of $\sim 1.72 \mathrm{~mJ} / \mathrm{cm}^{2}$ (approximately seven times lower than that of state-of-the-art designed heterostructure alloys, as reported by Fan F et al. (Nat. Nanotechnol. 10:796-803, 2015) considering the single pulse energy density) and degree of polarization $>99.9 \%$. Furthermore, the resulting three-colour single-mode lasing possesses the largest wavelength coverage of $\sim 186 \mathrm{~nm}$ (ranging from $\sim 534$ to $\sim 720 \mathrm{~nm}$ ) ever reported. These findings may open a new route to the exploitation of multicolour single-mode micro/nanolasers constructed by MOF engineering for photonic and biochemical applications.
\end{abstract}

\section{Introduction}

Multicolour single-mode polarized laser sensing/imaging is a very promising diagnostic technology that has yet to be developed ${ }^{1}$ (Supplementary Fig. 1). First, a light source with broadband (visible to near-infrared) multicolour output provides the fundamental basis for multimodal/multidimensional sensing/imaging ${ }^{2}$ since different tissues, cells or biochemicals have different responses (optical $^{3-5}$, thermal ${ }^{6-8}$, acoustic ${ }^{9,10}$, etc.) to different wavelengths of light. On the other hand, the polarization properties of light provide an opportunity for the analysis and processing of scattered light signals and can help

\footnotetext{
Correspondence: Guodong Qian (gdqian@zju.edu.cn)

${ }^{1}$ State Key Laboratory of Silicon Materials, Cyrus Tang Center for Sensor Materials and Applications, School of Materials Science and Engineering,

Zhejiang University, 310027 Hangzhou, China

${ }^{2}$ Department of Chemistry, University of Texas at San Antonio, San Antonio, TX 78249, USA

Full list of author information is available at the end of the article

These authors contributed equally: Huajun He, Yuanjing Cui
}

obtain structural information rich in biological materials $^{11,12}$. In addition, single-mode micro/nanolasers meet the application requirements of miniaturized photonic devices with high information accuracy, avoiding false signals and overlapping interference of different optical signals, with the potential to achieve target sensing/imaging of various cells and molecules when combined with multicolour output characteristics ${ }^{13,14}$. To utilize the uniqueness of broadband multicolour output, polarization and single-mode micro/nanolasing, monomeric multicolour polarized single-mode lasing micro/nanocrystals with controllable output from the visible to near-infrared (NIR) are potentially very useful for miniaturized multimodal sensing/imaging but have never been realized. To date, the use of semiconductor heterostructures with different gain bands has only achieved multicolour amplified spontaneous emission (ASE) or multimode lasing in the visible band without reports of polarization

\section{(c) The Author(s) 2020}

(c) (i) Open Access This article is licensed under a Creative Commons Attribution 4.0 International License, which permits use, sharing, adaptation, distribution and reproduction cc) in any medium or format, as long as you give appropriate credit to the original author(s) and the source, provide a link to the Creative Commons license, and indicate if changes were made. The images or other third party material in this article are included in the article's Creative Commons license, unless indicated otherwise in a credit line to the material. If material is not included in the article's Creative Commons license and your intended use is not permitted by statutory regulation or exceeds the permitted use, you will need to obtain permission directly from the copyright holder. To view a copy of this license, visit http://creativecommons.org/licenses/by/4.0/. 
properties, which was often accompanied by severe spontaneous emission components ${ }^{1,15-19}$.

Among various active luminescent materials, organic luminescent materials can combine simple processing techniques with molecular diversity and material compatibility and are considered one of best gain media for manufacturing multiwavelength lasers ranging from the visible to NIR, but adverse interactions between organic molecules, such as aggregation-caused quenching (ACQ) and energy transfer phenomena caused by Förster resonance, make it difficult to achieve efficient and stable multicolour laser output ${ }^{13,20}$. The metal-organic framework (MOF), a periodic porous crystalline material assembled by metal ions and organic bridging ligands, is considered a powerful hybrid material platform and provides opportunities to solve the mentioned issues ${ }^{21-25}$. Its highly engineered pore/channel structure provides excellent spatial confinement to assemble and isolate guest organic molecules, which can reduce intermolecular interactions for effective inhibition of excited-state energy transfer and even control of output emission characteristics $^{26-28}$. In addition, the smooth and regular crystal morphology of the MOF can enable it to effectively act as an optical resonator to provide optical feedback ${ }^{29}$. If guest organic molecules with different gain bands can be spatially separated by MOF crystal and pore/channel engineering while maintaining the efficient spatial confinement and orientation of the host MOF for the guest organic molecules, that is, ensuring an extremely high-gain concentration and oriented emission transition moment, then realization of a multicolour single-mode polarized laser with a low threshold in the shortened resonant cavity is expected.

Here, we demonstrate simultaneous in situ assembly of different guest dye molecules based on the homoepitaxy process of the host framework ZJU-68 ${ }^{26}$ in an optimized host-guest hierarchical hybrid MOF single crystal system to achieve multicolour single-mode lasing. The MOF hierarchical hybrid single crystal exhibits different macroscopically segmented colours, which suggests that spatially segmented assembly of different dye molecules in a single MOF microcrystal has been achieved. The homoepitaxial growth of the host framework ZJU-68 ensures uniformity and high quality of the single monolithic cavity. The spatially segmented assembled, tightly confined and highly oriented cationic linear dye molecules (E)-1-methyl-4-(2-(1-methyl-1H-pyrrol-2-yl)vinyl)pyr-

idinium (MMPVP), (E)-4-(4-(dimethylamino)styryl)-1methylpyridinium (DMASM) and 4-((1E,3E)-4-(4-(dimethylamino)phenyl)buta-1,3-dienyl)-1-methylpyridinium (DPBDM) in the anionic ZJU-68 sub-nanochannels efficiently minimize the intermolecular energy transfer (in particular avoiding the severe energy transfer caused by overlap of the short-wavelength dye emission band and the long-wavelength dye absorption band), increase the gain concentration, and optimize the orientation of dye molecules within the framework, resulting in high-gain dynamically controllable multicolour single-mode lasing with a degree of polarization $>99.9 \%$ and a low threecolour-lasing threshold of $\sim 1.72 \mathrm{~mJ} / \mathrm{cm}^{2}$ in a shortened laser cavity. Combined with the lasing pattern of the sample, the computationally simulated electric field distribution reveals the possible lasing mode mechanisms of the three colours of light waves in the hybrid cavity and qualitatively explains their propagation differences. In addition, the largest multicolour single-mode laser emission wavelength coverage in a single monolithic structure has been observed, with a wavelength range of $186 \mathrm{~nm}$ (from visible light $\sim 534$ to NIR $\sim 720 \mathrm{~nm}$ ). The results demonstrate the unique strategy of spatially controlling the laser colour through regional assembly of a guest gain medium constructed via MOF crystal engineering and provide a new route for multicolour solid-state microlaser materials for photonic integration and multimodal biochemical sensing/imaging.

\section{Results}

\section{Synthesis and characterization}

Figure 1 schematically shows the newly developed method for fabricating uniaxially homoepitaxially grown (UHG) ZJU-68 metal-organic framework crystals. The ZJU-68 crystal is constructed by the organic ligand 7-(4carboxyphenyl)quinoline-3-carboxylic acid $\left(\mathrm{H}_{2} \mathrm{CPQC}\right)$ and the trinuclear secondary building unit $(\mathrm{SBU})\left[\mathrm{Zn}_{3} \mathrm{O}\right]^{4}$ + formed by bridging $\mathrm{Zn}^{2+}$ and $\mu_{3}-\mathrm{O}^{26}$. Through the structural analysis of the host framework ZJU-68 (Fig. 1), it is known that the growth along the crystal axis is mainly ascribed to the coordination of the carboxyl groups at the two ends of the organic ligand with the SBU, while the growth in the radial direction mainly depends on the coordination of the nitrogen atom at the side end of the organic ligand with the SBU, whereby the axial coordination density is much higher than that in the radial direction from a spatial perspective. When the $\mathrm{pH}$ of the reaction solution is lowered, $\mathrm{H}^{+}$preferentially coordinates with the carboxyl groups at both ends of the ligand, thereby delaying the growth of the ZJU-68 crystal along the crystal axis direction, and the crystal tends to form massive particles. Conversely, elimination of the use of an additional acid is beneficial to the growth of the ZJU-68 crystal along the crystal axis, which not only contributes to shortening of the cavity length, facilitating the acquisition of single-mode lasing, but also effectively controls the ZJU-68 crystal tendency to homoepitaxially grow along the crystal axis direction. Furthermore, at the beginning of the preparation, the introduction of the substrate led to one end face of a considerable portion of the ZJU-68 crystals grown in the first step adhering to the 


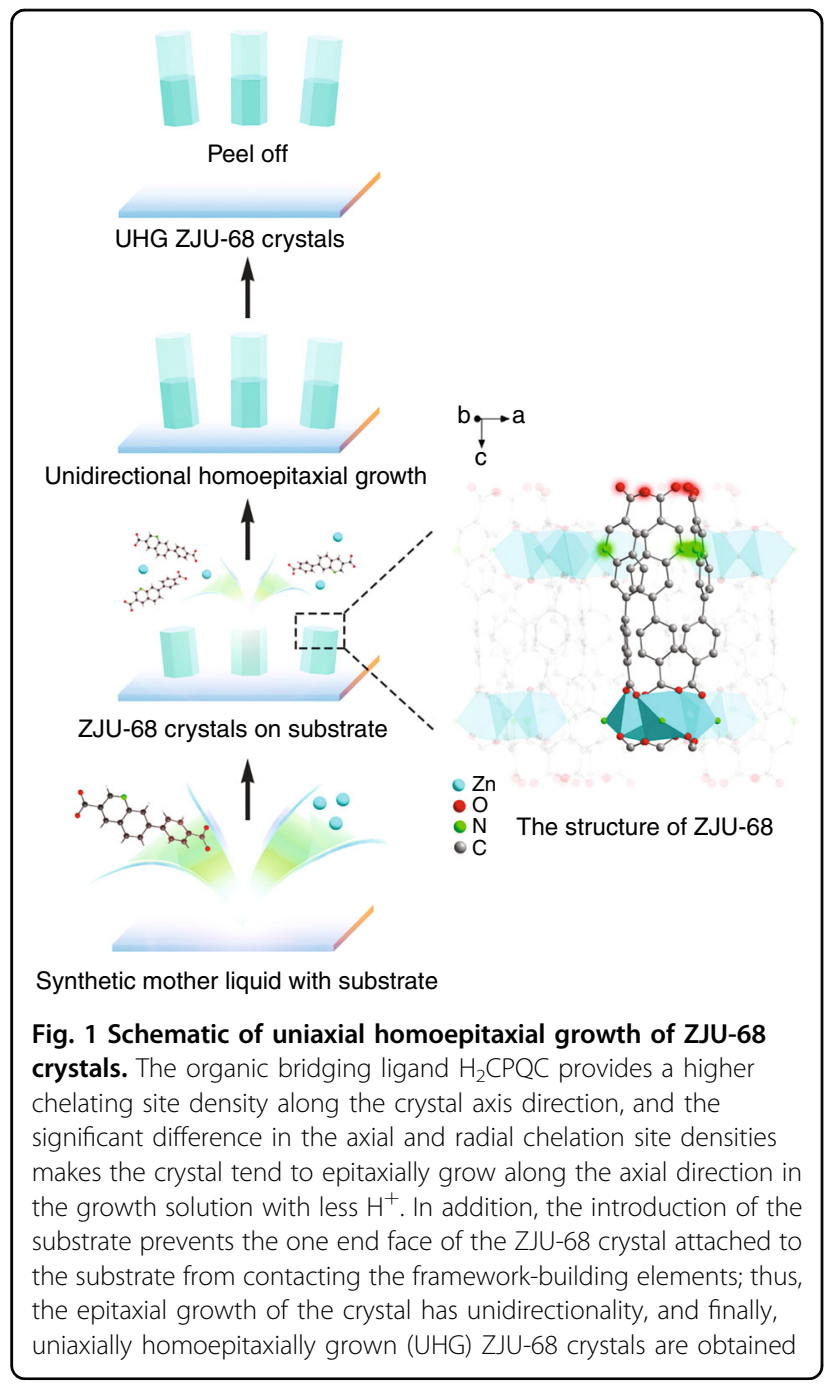

substrate, preventing it from contacting the frameworkconstructing elements in the subsequent homoepitaxial growth, thereby making the homoepitaxial growth of the crystal unidirectional instead of bidirectional, and finally, uniaxially homoepitaxially grown ZJU-68 crystals were obtained. Combined with the advantages of spatial confinement of MOF pores/channels, this approach of controlling the unidirectional homoepitaxial growth of MOF crystals brings about opportunities for spatially ordered assembly of different guest materials. As such, the dyeassembled hierarchical hybrid metal-organic framework structure was synthesized by the combination of in situ assembly and homoepitaxial growth mentioned above. The synthesis procedure is shown schematically in Fig. 2a. First, in situ self-assembly of $\mathrm{Zn}^{2+}$, organic linker $\mathrm{H}_{2} \mathrm{CPQC}$ and one kind of dye molecule (denoted dye-1) in $\mathrm{N}, \mathrm{N}$-dimethylformamide/acetonitrile at $100{ }^{\circ} \mathrm{C}$ afforded monochromatic hexagonal prism microcrystals ${ }^{26}$, denoted ZJU-68Jdye-1. Second, the resulting microcrystals were then immersed into a new reaction solution of $\mathrm{Zn}^{2+}$ and $\mathrm{H}_{2} \mathrm{CPQC}$ with another kind of dye molecule (denoted dye-2) to self-assemble two-colour hierarchical hybrid microcrystals, denoted ZJU-68Jdye-1+dye-2. Third, repeating the second step, three-colour or multicolour hierarchical hybrid microcrystals ZJU-68つdye-1+dye$2+\cdots+$ dye $n$ could be obtained (dye- $n$ denotes the dye molecule used in the $n^{\text {th }}$ step). Three different linear dye molecules were used in this study, namely, $(E)-1$ methyl-4-(2-(1-methyl-1H-pyrrol-2-yl)vinyl)pyridinium (MMPVP), (E)-4-(4-(dimethylamino)styryl)-1-methylpyridinium (DMASM) and 4-((1E,3E)-4-(4-(dimethylamino) phenyl)buta-1,3-dienyl)-1-methylpyridinium (DPBDM). Through the combination of these three different dye molecules, seven different hybrid MOF single crystals could be obtained (Fig. 2c-i). These hybrid single crystals all have a hexagonal prismatic crystal morphology similar to that of pure ZJU-68 (Fig. 2b), except for the colour change due to the assembly of the dye molecules. The colours corresponding to the crystal segments in which the dyes MMPVP, DMASM, and DPBDM are assembled are light yellow, magenta, and purple, respectively. The assynthesized dye-assembled ZJU-68 microcrystals show magenta-yellow/purple-magenta/purple-yellow twosegment (Fig. 2f-h) and purple-magenta-yellow threesegment hierarchical structures (Fig. 2i) under bright field, confirming the successful in situ assembly and homoepitaxial growth of the MOFs.

Figure $2 \mathrm{j}$ shows the powder X-ray diffraction (PXRD) patterns of dye-assembled ZJU-68 hierarchical hybrid microcrystals. The results show that the PXRD patterns of these hybrid crystals have the same peak positions as the simulated PXRD spectrum of pure ZJU- $68^{26}$, and there is no redundant peak, indicating that the structures of the as-synthesized hybrid crystals are pure and that the host ZJU-68 framework still maintains the initial crystal structure after assembly of different dye molecules and homoepitaxial growth. Since a fresh and concentrationdetermined solution is used for the homoepitaxial growth of each new crystal segment, the assembly concentrations of the dyes in the ZJU-68 crystal can be further determined by separately synthesizing monochromatic hybrid crystals of the specific dye molecules. Accordingly, as calculated by ${ }^{1} \mathrm{H}$ NMR analysis $(\mathrm{NMR}=$ nuclear magnetic resonance), per gram, the resulting ZJU-68DMMPVP crystals contain $80.8 \mathrm{mg}$ of dye molecules, corresponding to a concentration of $0.67 \mathrm{M}$ (the molar amount of dye per unit volume of the solid crystal) (Supplementary Fig. 2), which results in the corresponding chemical formula of the ZJU-68DMMPVP segment being (MMPVP $)_{0.48} \mathrm{H}_{1.52}\left[\mathrm{Zn}_{3} \mathrm{O}(\mathrm{CPQC})_{3}\right]$. The dye assembly concentrations of ZJU-68DDMASM and ZJU-68כDPBDM can be obtained by the same method (Supplementary Fig. 3 and Supplementary Fig. 4), which 

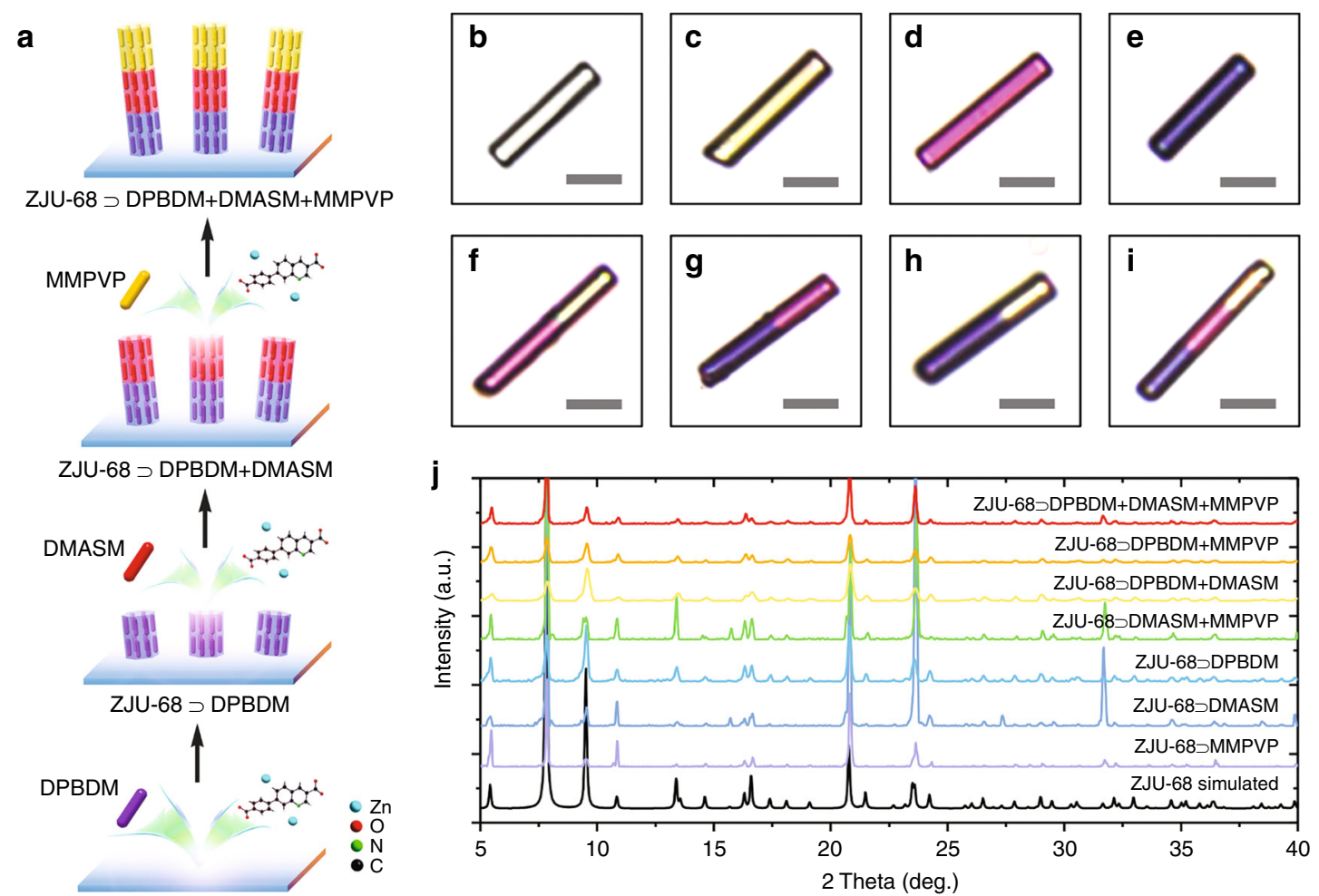

Fig. 2 Synthesis and characterization of UHG hierarchical hybrid ZJU-68 microcrystals. a Schematic of the synthesis of UHG hierarchical dyeassembled hybrid ZJU-68 microcrystals. b-i Optical micrographs of ZJU-68 (b), ZJU-68כMMPVP (c), ZJU-68כDMASM (d), ZJU-68כDPBDM (e), ZJU68JDMASM + MMPVP (f), ZJU-68DDPBDM + DMASM (g), ZJU-68DDPBDM + MMPVP (h), and ZJU-68DDPBDM + DMASM + MMPVP (i); scale bars, $10 \mu \mathrm{m}$. j PXRD patterns of ZJU-68 and hierarchical hybrid ZJU-68 microcrystals, which indicate that hierarchical hybrid ZJU-68 microcrystals have a framework structure identical to that of ZJU-68

are $\sim 0.44 \mathrm{M}$ and $\sim 0.53 \mathrm{M}$, and the corresponding chemical formulas are $(\text { DMASM })_{0.32} \mathrm{H}_{1.68}\left[\mathrm{Zn}_{3} \mathrm{O}(\mathrm{CPQC})_{3}\right]$ and $(\mathrm{DPBDM})_{0.38} \mathrm{H}_{1.62}\left[\mathrm{Zn}_{3} \mathrm{O}(\mathrm{CPQC})_{3}\right]$, respectively. Homoepitaxy does not require consideration of matching of the lattice parameters and is conducive to ensuring the consistency of the crystal growth direction before and after the epitaxy process, which means that the direction of the one-dimensional sub-nanochannels of ZJU-68 is always along the crystal axis. The width of the linear guest dye molecules used in the study is matched to the channel diameter of ZJU-68, and they all have a single absorption/ emission transition moment, which is oriented along the length of the molecule. Therefore, through the homoepitaxial in situ self-assembly strategy using the host framework ZJU-68, different linear dye molecules can be hierarchically assembled in ZJU-68 along the same direction, forming an oriented and hierarchical guest molecular integration system.

\section{Multicolour fluorescence}

Figure 3 compares the photoluminescence (PL) spectra of dye-assembled ZJU-68 hybrid crystals. It can be seen from the characteristics of the PL spectra that after assembly of the dye molecules, the fluorescence emission from the crystals is entirely converted from the blue-light emission of pure ZJU-68 (peak at $\sim 450 \mathrm{~nm}$, see Fig. 3a) to the characteristic emission colours of the dyes. Under excitation by a mercury lamp with a $480 \mathrm{~nm}$ excitation filter set, ZJU-68כMMPVP, ZJU-68כDMASM and ZJU$68 \supset D P B D M$ show green, red, and near-infrared emission with peaks at $\sim 540, \sim 620$, and $\sim 716 \mathrm{~nm}$, respectively (Fig. 3b-d). The corresponding full widths at half maximum (FWHMs) are 70.5, 54.7, and $61.0 \mathrm{~nm}$. The reasons for choosing $480 \mathrm{~nm}$ as the excitation wavelength are as follows: (1) The optimal absorption position of the dye DMASM is near $470 \mathrm{~nm}^{30}$, and the remaining two dye molecules can be effectively excited by $480 \mathrm{~nm}^{31}$. (2) The $480 \mathrm{~nm}$ wavelength light is located in the emission band of ZJU-68 and is basically not absorbed by ZJU-68 (see Supplementary Fig. 5), which is conducive to promoting the population inversion process of the dye molecules by avoiding the optical loss caused by the hostguest energy transfer process. (3) Compared with ultraviolet light, $480 \mathrm{~nm}$ light causes less damage to organic molecules, which is conducive to the stability of the resulting luminescence performance. For the 


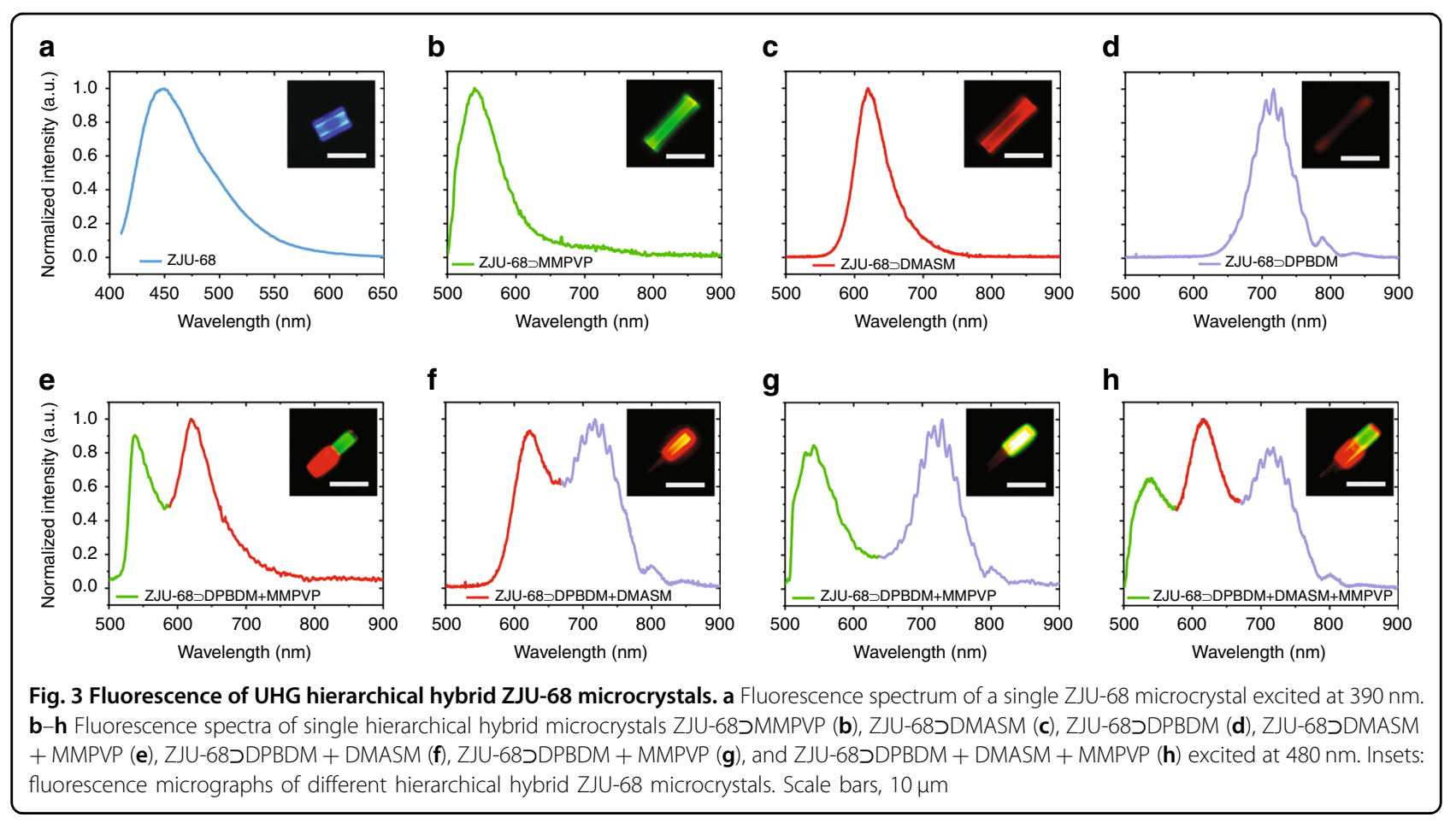

homoepitaxial hybrid MOF structures in which different dye molecules are assembled in different crystal segments, the emission of different colours is spatially segmented under excitation at $480 \mathrm{~nm}$ (Fig. 3e-h). The emission peak positions of these different colour segments are substantially coincident with the emission peak positions of the MOF structures assembled by the monochromatic dyes. Taking ZJU-68DDPBDM + DMASM + MMPVP as an example (Fig. 3h), the fluorescence emission spectrum has three peak positions, namely, 540, 619, and $716 \mathrm{~nm}$, corresponding to the three-segment assembly of MMPVP, DMASM, and DPBDM, and there is no significant peak shift phenomenon. It is worth noting that for the hybrid crystals shown in Fig. 3, when the emission detection direction is perpendicular to the crystal axis, almost no fluorescence signal can be observed upon excitation. This is because the MMPVP, DMASM, and DPBDM linear molecules can be orderly aligned in the interior of the framework by utilizing the size-matched one-dimensional channels of ZJU- $68^{26,28}$. The alignment of the emission transitions of these dye molecules thus results in significant emission anisotropy. Multichannel confocal laser scanning microscopy also clearly shows that the hybrid single crystal ZJU-68DDPBDM + DMASM + MMPVP can be combined with different incident light and filter modules to obtain segmented excitation and different colour fluorescence signal output (Supplementary Fig. 6). The definite segmented fluorescence boundaries indicate that the ZJU-68 homoepitaxial crystal is substantially grown along the crystal axis direction, which is different from the core-shell epitaxial growth of most MOF crys$\mathrm{tals}^{32,33}$. This should be mainly due to the number and density of coordination sites in the axial direction of the ZJU-68 structure being much larger than those in the radial direction. Through the combination of selfassembly and homoepitaxial methods, the advantages of efficient spatial confinement and spatial segmentation of different dye molecules provided by ZJU-68 are successfully integrated, effectively avoiding the aggregationcaused quenching (ACQ) effect and the energy transfer from short-wavelength dye molecules to long-wavelength dye molecules, which is conducive to efficient multiwavelength emission output.

\section{Multicolour lasing performance}

The lasing property was investigated for an individual small hybrid crystal of ZJU-68 DDPBDM + DMASM + MMPVP with a side length $(R)$ of $\sim 1.65 \mu \mathrm{m}$ under a microscope at room temperature. A 480-nm laser beam from a femtosecond optical parametric amplifier (OPA) was coupled into the microscope. The PL signal of the test sample was collected by a fibre optic spectrometer. Figure 4 shows the representative PL spectra of the isolated hybrid crystal near the lasing threshold. The hierarchical hybrid single crystal ZJU-68DDPBDM + DMASM + MMPVP is divided into three sections, which correspond to the ZJU$68 \supset D P B D M$ crystal segment, ZJU-68DDMASM segment, and ZJU-68DMMPVP segment. For the crystal segment in which the DPBDM molecule is assembled (Fig. 4a), the fibre optic spectrometer shows no substantial 

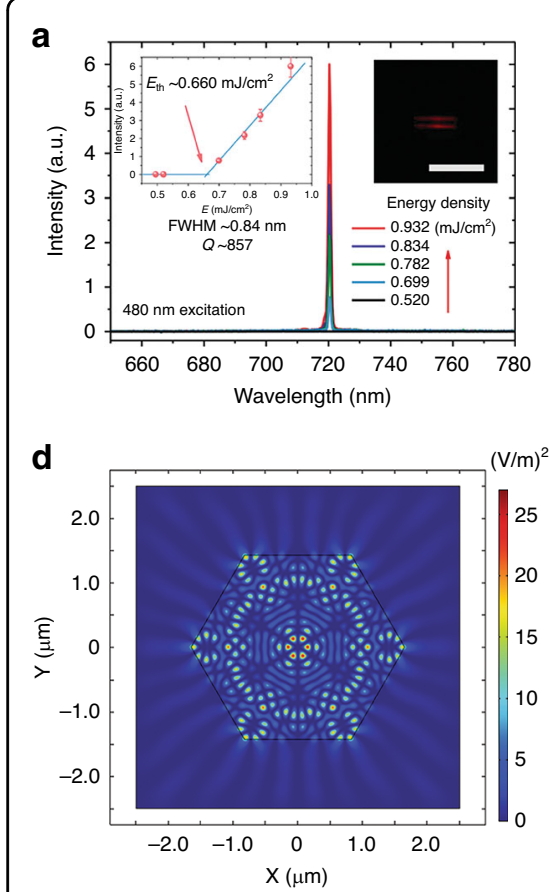
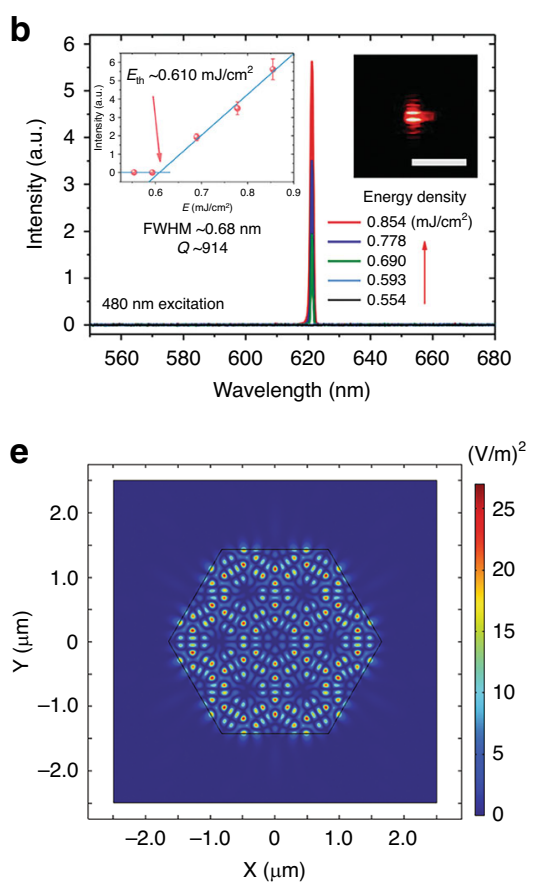
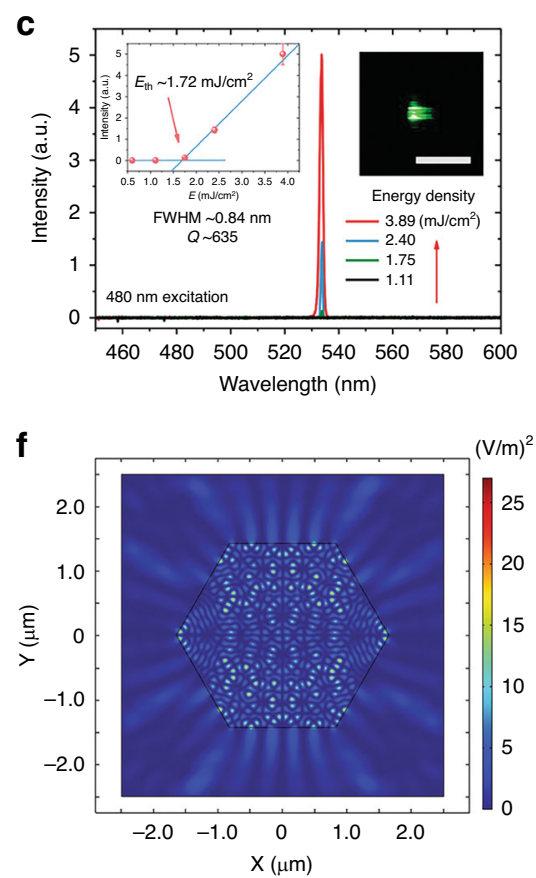

Fig. 4 Single-mode lasing in an individual ZJU-68DDPBDM + DMASM + MMPVP hierarchical hybrid microcrystal $(R \sim 1.65 \mu \mathrm{m})$. a-c Emission spectra of ZJU-68DDPBDM (a), ZJU-68DDMASM (b), and ZJU-68JMMPVP (c) crystal segments around the lasing threshold. Insets: micrographs of ZJU-68JDPBDM (a), ZJU-68JDMASM (b) and ZJU-68כMMPVP (c) crystal segments excited at $480 \mathrm{~nm}$ (right), and emission intensity as a function of pump fluence showing a lasing threshold of $\sim 0.660 \mathrm{~mJ} / \mathrm{cm}^{2}$ for ZJU-68DDPBDM, $\sim 0.610 \mathrm{~mJ} / \mathrm{cm}^{2}$ for ZJU-68DDMASM, and $\sim 1.72 \mathrm{~mJ} / \mathrm{cm}^{2}$ for ZJU68JMMPVP (left). Scale bars, $10 \mu \mathrm{m}$. d-f Simulated electric field distributions (square of the electric field intensity) in the hexagonal cavity for the $720 \mathrm{~nm}(\mathbf{d}), 621 \mathrm{~nm}(\mathbf{e})$, and $534 \mathrm{~nm}(\mathbf{f})$ modes

luminescence signal under excitation at a low pump fluence $(E)$ of $\leq 0.520 \mathrm{~mJ} / \mathrm{cm}^{2}$. The pump fluence is defined as the laser energy density directly received by the MOF crystal (after going through the objective lens and before being incident on the MOF crystal). As the pump energy density increases to $\geq 0.699 \mathrm{~mJ} / \mathrm{cm}^{2}$, a narrow peak centred at $\sim 720 \mathrm{~nm}$ appears and increases rapidly as the pump fluence increases, while the intensity of the broad spontaneous emission remains almost constant. The crystal photomicrograph taken during the pumping process (the right inset of Fig. 4a) shows bright dark-red striped patterns on both sides of the hexagonal prism crystal, which can be ascribed to the whispering gallery mode (WGM) mechanism as discussed in our previous work $^{28}$. The left inset in Fig. 4a illustrates the pump fluence dependence of the emission intensity, indicating a linear relationship with the pump fluence and a low lasing threshold of $E_{\mathrm{th}} \sim 0.660 \mathrm{~mJ} / \mathrm{cm}^{2}$. The full width at half maximum (FWHM) of the pinpoint narrow peak is $\sim 0.84 \mathrm{~nm}$, which is two orders of magnitude narrower than that $(61.0 \mathrm{~nm})$ of the fluorescent emission band of ZJU-68DDPBDM. The presence of a single peak accompanied by a significant spectral narrowing and the threshold energy coupled with the linear rapid increase in intensity with the pump fluence suggest that single-mode lasing has occurred in the ZJU-68DDPBDM crystal segment. The quality factor $(Q)$ is given by $Q=\lambda / \delta \lambda$, where $\lambda$ and $\delta \lambda$ are the peak wavelength and FWHM, respectively. At a pump fluence of $0.834 \mathrm{~mJ} / \mathrm{cm}^{2}$, the FWHM of the lasing peak at $720 \mathrm{~nm}$ is $\sim 0.84 \mathrm{~nm}$, and the crystal segment can be deduced to have a quality factor of $Q \sim 857$ for the laser cavity. The crystal segment ZJU68DDMASM (Fig. 4b) or ZJU-68כMMPVP (Fig. 4c) assembled with DMASM or MMPVP molecules can also achieve single-mode lasing operation, showing a bright red or bright green stripe-spot pattern with the spike-like spectrum peaking at $621 \mathrm{~nm}$ or $534 \mathrm{~nm}$ during pumping. The emission intensity as a function of pump fluence shows that their lasing thresholds are $\sim 0.610 \mathrm{~mJ} / \mathrm{cm}^{2}$ and $\sim 1.72 \mathrm{~mJ} / \mathrm{cm}^{2}$, and the quality factors $Q$ are calculated as $\sim 914$ and $\sim 635$, respectively. Compared to the crystal segments in which DMASM or DPBDM are assembled, the higher threshold and lower $Q$ of the ZJU-68כMMPVP crystal segment may be attributed to the weaker lasing efficiency of MMPVP resulting in a higher pump energy density, and the high-energy photons at a high pump fluence will affect the quality of the cavity. Although the results show that the three-colour lasing can be attributed 


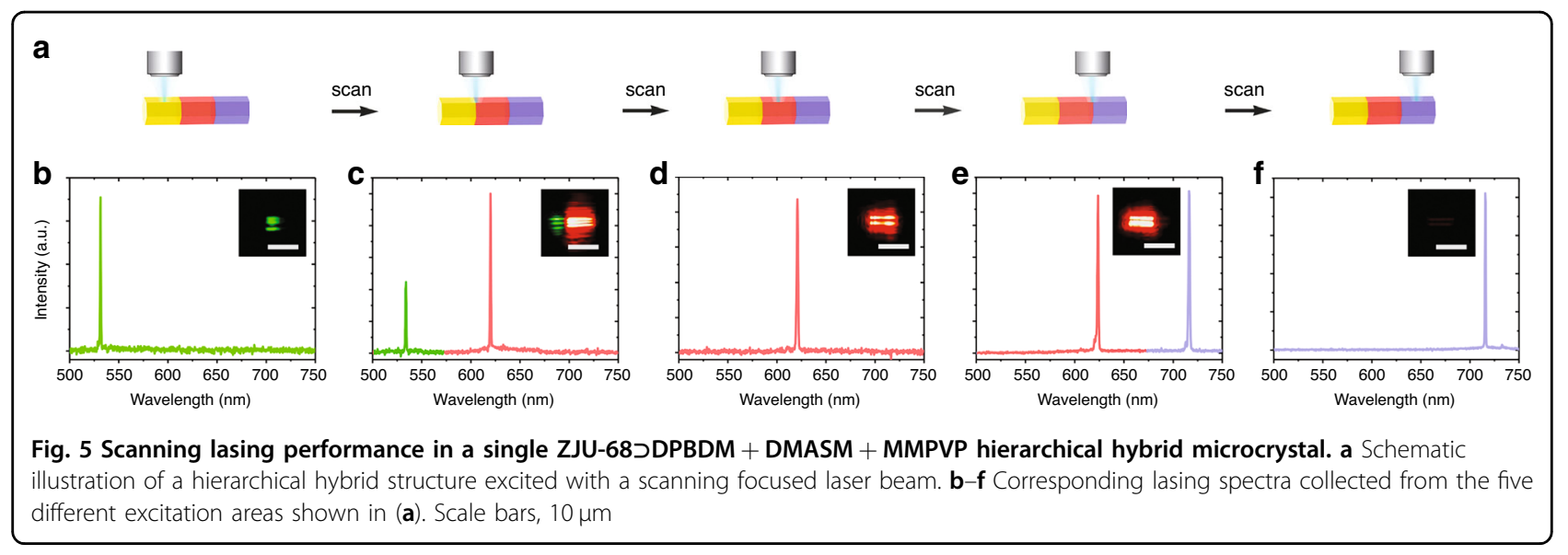

to the WGM mechanism, basically the same cavity will have different effects on the propagation and feedback of different wavelengths within it. To reveal these possible differences and the more detailed lasing mode mechanisms in the hierarchical hybrid hexagonal cavity, qualitative optical simulations were further conducted in an eigenfrequency study by using COMSOL Multiphysics (version 5.4). Figure $4 \mathrm{~d}-\mathrm{f}$ shows the computed electric field distributions (square of the electric field intensity) in the hierarchical hybrid hexagonal cavity for analysed mode wavelengths of 720,621 , and $534 \mathrm{~nm}$. Compared with the distribution of red light $(621 \mathrm{~nm})$ and green light $(534 \mathrm{~nm})$, the distribution of near-infrared light $(720 \mathrm{~nm})$ in the cavity is more concentrated at the corners of the hexagonal cavity, which matches the lasing pattern in optical images (insets in Fig. $4 \mathrm{a}-\mathrm{c}$ ) very well. For the crystal (cavity) size used in the experiment $(R=1.65 \mu \mathrm{m})$, red light with a wavelength of $621 \mathrm{~nm}$ shows a more concentrated and stronger optical field distribution, while the simulation result corresponding to $534 \mathrm{~nm}$ green light is the most disperse and weak. In addition, the characteristics of the WGM mechanism, that is, the internal reflection of the six crystal facets in turn, can be clearly seen in the simulated diagrams for the NIR lasing at $720 \mathrm{~nm}$ (Fig. 4d) and the red lasing at $621 \mathrm{~nm}$ (Fig. 4e). However, the simulation diagram for the green lasing at $534 \mathrm{~nm}$ (Fig. 4f) shows few such characteristics, indicating that the WGM mechanism has not been steadily established in this case. These results suggest that the cavity is more conducive to the formation of a stable standing wave mode for red light at $621 \mathrm{~nm}$ and NIR light at $720 \mathrm{~nm}$ such that the light loss is lower and the $Q$ value is greater, contributing to the lower thresholds, while the result for green light is the opposite, which corresponds to the experimental data. Considering the single pulse energy density, the three-colour-lasing threshold of the resulting ZJU-68DDPBDM + DMASM + MMPVP hybrid single crystal is $\sim 1.72 \mathrm{~mJ} / \mathrm{cm}^{2}$, which is $\sim 7$ times lower than that of the state-of-the-art designed heterostructure alloy $\mathrm{ZnCdSSe}$ for three-colour lasing reported previously $^{1}$. A comparison of the lasing thresholds of multicolour lasing monomer materials reported thus far ${ }^{1,15-17,19,27,31,34}$ can be found in Supplementary Table 1. Although the threshold has a relatively high peak power density compared with some reported multicolour lasing monomer materials due to the narrow excitation pulse width, it is foreseeable that the lasing threshold of the resulting ZJU-68DDPBDM + DMASM + MMPVP can be further reduced by means such as thin metal layer coating $^{35}$. Combining the advantages of a wide and controllable emission range, a single mode, a narrow line width and high linear polarization output (see details below), the resulting ZJU-68DDPBDM + DMASM + MMPVP still has high competitiveness compared with existing monolithic multicolour lasing materials (see Supplementary Table 2). In addition, most of the reported multicolour lasing was still accompanied by obvious or severe spontaneous emission components ${ }^{1,16,17}$, which indicates the difficulty of constructing an excellent cavity for multicolour lasing to a certain degree. The highquality factor, acceptably low multicolour lasing threshold and highly polarized emission with almost pure stimulated emission of ZJU-68DDPBDM + DMASM + MMPVP should be attributed to the efficient spatial confinement effect provided by host metal-organic framework ZJU-68 and the fact that the smooth and regular crystal morphology of ZJU-68 is maintained while assembling different dye molecules during the epitaxial growth process.

The MOF homoepitaxial structure obtained by hierarchically assembling different dye molecules provides the opportunity for scanning multicolour lasing. Taking the ZJU-68DDPBDM + DMASM + MMPVP single microcrystal as an example, Fig. 5a illustrates the scanning pump lasing in different regions of the microcrystal along the crystal axis direction. Each part of the hierarchical 

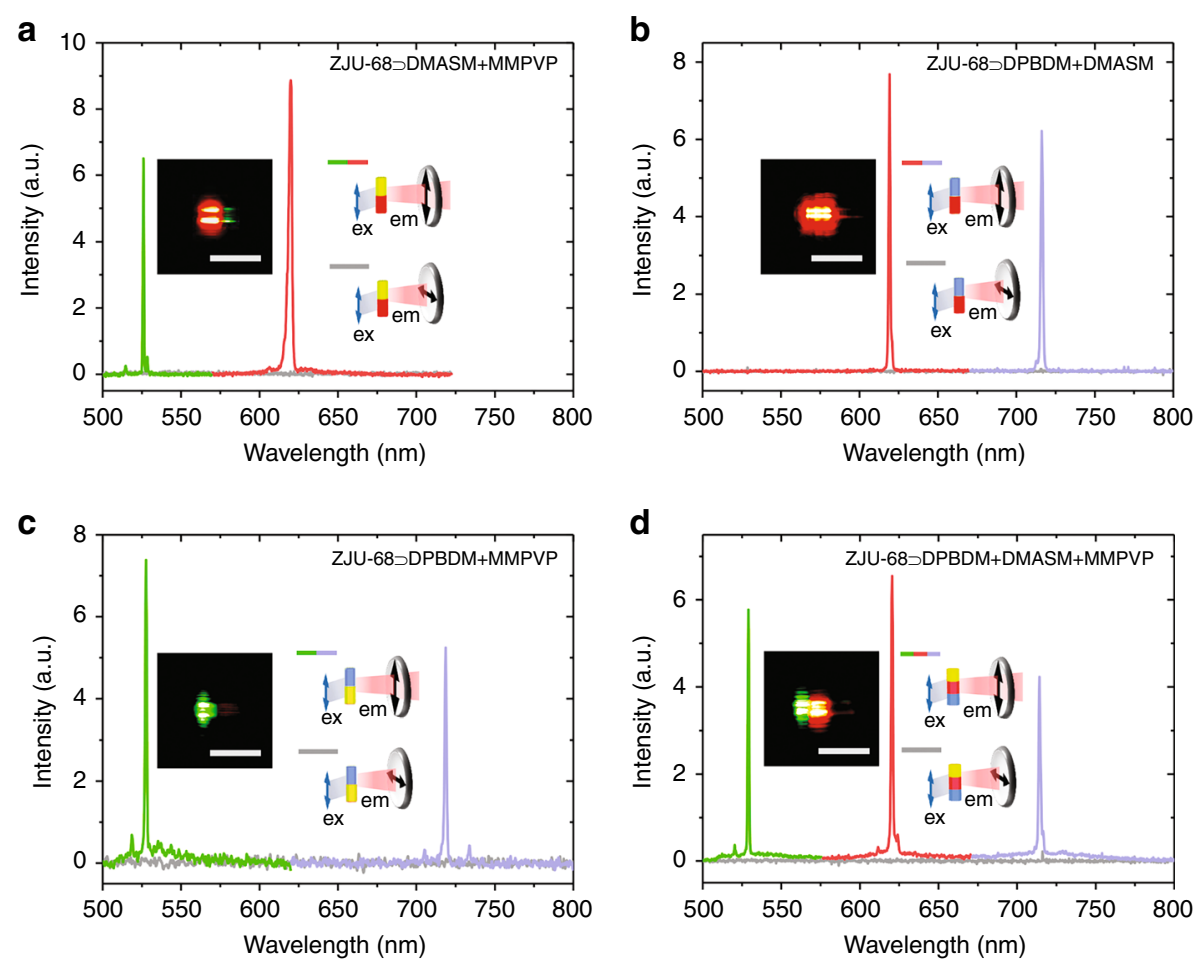

Fig. 6 Tuneable multicolour polarized lasing performance in hierarchical hybrid ZJU-68 microcrystals. a-d Intensity-dependent emission spectra from individual hierarchical hybrid microcrystals ZJU-68DDMASM + MMPVP (a), ZJU-68כDPBDM + DMASM (b), ZJU-68DDPBDM + MMPVP (c), and ZJU-68DDPBDM + DMASM + MMPVP (d) with the emission detected at two polarization angles of $\theta=0^{\circ}$ (parallel to the crystal channels) and $\theta=90^{\circ}$ (perpendicular to the crystal channels), excited at $480 \mathrm{~nm}$. Insets: Schematic diagrams of the measurement geometry for an individual crystal, and micrographs of hierarchical hybrid ZJU-68 microcrystals excited at $480 \mathrm{~nm}$. Scale bars, $10 \mu \mathrm{m}$

structure is selectively focused on and excited by a femtosecond laser $(480 \mathrm{~nm})$ that passes through an objective lens, and the corresponding lasing spectra are shown in Fig. $5 \mathrm{~b}-\mathrm{f}$. The excitation position is initially at one end of the hybrid crystal, passes through the junction of two crystal segments, then proceeds to the next segment, and so on. Each crystal segment exhibits a sharp single peak at approximately 531 (Fig. 5b), 620 (Fig. 5d), and $716 \mathrm{~nm}$ (Fig. 5f) upon excitation, corresponding to green, red, and near-infrared (NIR) emission. When the pump position is at the junction of crystal segments, the two crystal segments are simultaneously excited to obtain bright green/ red (Fig. 5c) or red/NIR lasing (Fig. 5e), corresponding to the emission spectrum of the two single peaks, and the peak positions have no obvious displacement. The unique structure obtained by hierarchically assembling different dye molecules allows a laser of a specific colour or a combination of colours to be controllably outputted in a micro-nanospace, potentially for use in many fields, such as flow biochemical sensing and on-chip optical signal transmission and processing.

Figure 6 shows the anisotropic lasing performance of dyeassembled hierarchical hybrid crystals, where the polarization direction of the pump light $(480 \mathrm{~nm}, 2.01 \mathrm{~mJ} /$ $\mathrm{cm}^{2}$ ) is fixed direction parallel to the crystal axis. The PL signal from the sample first passes through a polarizer and then is collected by a fibre optic spectrometer. When the emission detection polarization direction is parallel to the crystal

axis, the two-colour hierarchical hybrid crystals ZJU68DDMASM + MMPVP (Fig. 6a), ZJU-68כDPBDM + DMASM (Fig. 6b), and ZJU-68DDPBDM + MMPVP (Fig. 6c) display dual-wavelength single-mode lasing spectra, accompanied by intense red/green, red/dark-red, and green/dark-red stripe-spot patterns, respectively. Among them, the single-mode lasing peak corresponding to the green light is located near $530 \mathrm{~nm}$, the red light peak is at $\sim 620 \mathrm{~nm}$, and the dark-red light (near-infrared) peak is at $\sim 716 \mathrm{~nm}$. When the emission detection polarization direction is perpendicular to the crystal axis, a light emission signal can hardly be observed (consistent with the case of the monochromatic hybrid crystal ZJU-68DMMPVP, ZJU-68DDMASM, or ZJU-68כDPBDM, see Supplementary Fig. 7), which indicates that the emission has strong polarization, and the polarization direction is substantially oriented along the crystal axis. It is worth emphasizing that 
three-wavelength single-mode lasing is successfully realized in the three-colour hierarchical hybrid crystal ZJU$68 \mathrm{DPBDM}+\mathrm{DMASM}+\mathrm{MMPVP}$, and the single-mode lasing peak positions have no obvious displacement compared with the above data. Significant lasing light polarization is also observed in this system (Fig. 6d). The degree of polarization can be defined as DOP $=\left(I_{\|}-I_{\perp}\right) /\left(I_{\|}+I_{\perp}\right)$ in our experiments $\left(I_{\|}\right.$or $I_{\perp}$ denotes the emission intensity detected when the emission detection polarization direction is parallel or perpendicular to the crystal axis, respectively) ${ }^{35}$, and the DOP of the above multicolour singlemode lasing can be calculated to be $>99.9 \%$ (limited by the spectrometer sensitivity of our measurement system), demonstrating the first example of an inherently polarized multicolour lasing microcrystalline material. In addition, when the polarization direction of the pump light is perpendicular to the crystal axis, the dye-loaded ZJU-68 microcrystal can hardly be excited effectively; therefore, nearly no emission signals can be observed. The sensitive response to the excitation light polarization as well as inherently polarized lasing is attributed to the highly oriented assembly of the linear dye molecules within the one-dimensional channels of the host framework ZJU-68 microcrystal given that the absorption/emission transition moments of the linear dye molecules MMPVP, DMASM, and DPBDM are basically oriented along the molecular axis. These anisotropic multicolour lasing results indicate great potential for biochemical sensing/imaging, as well as optical signal processing in optoelectronic/photonic integration.

\section{Discussion}

In summary, we have reported hierarchical assembly of different dye molecules based on a homoepitaxy process in a host-guest hybrid MOF microresonator to achieve up to three-wavelength single-mode lasing. An isomorphic cavity system with optimized segmented multicolour gain in axial space is constructed by simultaneously assembling different dye molecules in situ with the homoepitaxial host framework ZJU-68. The segmented assembly of different dye molecules not only spatially manipulates the colour output of the microlaser but also effectively solves the adverse effects of energy transfer between different dye molecules, especially for the systems with strong energy transfer in which the emission band of a shortwavelength dye substantially overlaps with the absorption band of a long-wavelength dye, thereby broadening the output band of the dye-based multicolour microlaser. In addition, owing to the high-efficiency spatial confinement effect and the regular smooth crystal morphology provided by ZJU-68, enabling the system to act as a highquality resonator, the resulting three-colour lasing exhibits perfectly polarized emission, a low lasing threshold (single pulse energy density), a high-quality factor and almost pure stimulated emission compared to existing single monolithic materials for multicolour lasing. Furthermore, three-colour single-mode lasing over a wavelength range of $186 \mathrm{~nm}$ (from visible $\sim 534 \mathrm{~nm}$ to NIR $\sim 720 \mathrm{~nm}$ ) in a single monolithic structure, the largest ever reported, has been observed. Our work greatly simplifies the process of creating a monolithic single-mode laser structure with dynamically controllable emission from the visible to NIR. It is foreseeable that these results will provide a reference for multimodal biochemical sensing/ imaging and on-chip photonic information processing.

\section{Materials and methods}

\section{Synthesis of ZJU-68DDPBDM + DMASM + MMPVP}

A mixture of DPBDM iodide $(5.44 \mu \mathrm{mol}, \sim 2.1 \mathrm{mg}), \mathrm{Zn}$ $\left(\mathrm{BF}_{4}\right)_{2} \cdot \mathrm{xH}_{2} \mathrm{O} \quad(0.068 \mathrm{mmol}, \quad \sim 20 \mathrm{mg}), \quad \mathrm{H}_{2} \mathrm{CPQC}$ (0.034 mmol, $10 \mathrm{mg}), \mathrm{DMF}(2 \mathrm{~mL}), \mathrm{MeCN}(0.4 \mathrm{~mL})$, and $\mathrm{H}_{2} \mathrm{O}(0.01 \mathrm{~mL})$ was sealed in a $15 \mathrm{~mL}$ Teflon-lined stainless-steel bomb, subjected to ultrasonic vibration for $5 \mathrm{~min}$, and then heated at $100{ }^{\circ} \mathrm{C}$ for $24 \mathrm{~h}$. After cooling to room temperature, the reacted solution was carefully removed, the purple crystals were washed three times with fresh DMF, and the DMF wash was thoroughly removed. A mixture of DMASM iodide $(5.44 \mu \mathrm{mol}$, $\sim 2.0 \mathrm{mg}), \mathrm{Zn}\left(\mathrm{BF}_{4}\right)_{2} \cdot \mathrm{xH}_{2} \mathrm{O}(0.068 \mathrm{mmol}, 20 \mathrm{mg}), \mathrm{H}_{2} \mathrm{CPQC}$ $(0.034 \mathrm{mmol}, 10 \mathrm{mg}), \mathrm{DMF}(2 \mathrm{~mL}), \mathrm{MeCN}(0.4 \mathrm{~mL})$, and $\mathrm{H}_{2} \mathrm{O}(0.01 \mathrm{~mL})$ was sealed in the above Teflon-lined stainless-steel bomb containing the washed purple crystal product, which was then heated at $100^{\circ} \mathrm{C}$ for $6 \mathrm{~h}$. After cooling to room temperature, the reacted solution was carefully removed, the purple-red crystals were washed three times with fresh DMF, and the DMF wash was thoroughly removed. A mixture of MMPVP iodide $(5.44 \mu \mathrm{mol}, \quad \sim 1.8 \mathrm{mg}), \quad \mathrm{Zn}\left(\mathrm{BF}_{4}\right)_{2} \cdot \mathrm{xH}_{2} \mathrm{O} \quad(0.068 \mathrm{mmol}$, $\sim 20 \mathrm{mg}), \mathrm{H}_{2} \mathrm{CPQC}(0.034 \mathrm{mmol}, 10 \mathrm{mg})$, DMF $(2 \mathrm{~mL})$, $\mathrm{MeCN}(0.4 \mathrm{~mL})$, and $\mathrm{H}_{2} \mathrm{O}(0.01 \mathrm{~mL})$ was sealed in the above Teflon-lined stainless-steel bomb containing the washed purple-red crystal product, which was then heated at $100^{\circ} \mathrm{C}$ for $6 \mathrm{~h}$. After cooling to room temperature and decanting the mother liquor, the purple-red-yellow segmented hexagonal crystalline product was rinsed four times with fresh DMF $(5 \mathrm{~mL} \times 4)$ and dried in air.

\section{Measurements}

For lasing experiments, an optical parametric amplifier (Spirit-OPA + Spirit-OPA-UV3, Newport Corporation) was pumped by a fully automated ultrafast laser system (Spirit One $1040-8,8 \mathrm{~W}$ at $1040 \mathrm{~nm}$, Newport Corporation), which was used for generating the excitation pulse $(1 \mathrm{kHz}$, $480 \mathrm{~nm}$, pulse width $<400 \mathrm{fs}$ ). The incident laser was coupled to a microscope (IX71, Olympus) for focusing on crystals through an objective lens (Olympus LUCPlanFL N $\times 40$, numerical aperture $=0.60$ ). The PL signal from the sample was then focused and collected by a fibre optic spectrometer (PG2000-Pro, Ideaoptics Instruments). 


\section{Acknowledgements}

We acknowledge the financial support from the National Natural Science Foundation of China (Nos. 51632008, U1609219, and 61721005), partial financial support from the National Science Foundation of the United States (1407443), and the Welch Foundation (AX-1730).

\section{Author details}

${ }^{1}$ State Key Laboratory of Silicon Materials, Cyrus Tang Center for Sensor Materials and Applications, School of Materials Science and Engineering, Zhejiang University, 310027 Hangzhou, China. ${ }^{2}$ Department of Chemistry, University of Texas at San Antonio, San Antonio, TX 78249, USA. ${ }^{3}$ Present address: Present address: Division of Physics and Applied Physics, School of Physical and Mathematical Sciences, Nanyang Technological University, 21 Nanyang Link, Singapore 637371, Singapore

\section{Author contributions}

H.H. conceived the idea and designed the experiments. H.H. synthesized the materials, performed the spectra experiments, and computationally simulated the electric field distributions of the cavity. H.L. and K.S. assisted with synthesis of dye molecules and ${ }^{1} \mathrm{H}$ NMR characterization. H.H. wrote the first draft of the manuscript. Y.C., B.C., and G.Q. participated in data analysis and provided major revisions. All authors discussed the results and commented on the manuscript.

\section{Conflict of interest}

The authors declare that they have no conflict of interest.

Supplementary information is available for this paper at https://doi.org/ 10.1038/s41377-020-00376-7.

Received: 10 March 2020 Revised: 18 July 2020 Accepted: 28 July 2020 Published online: 13 August 2020

\section{References}

1. Fan, F. et al. A monolithic white laser. Nat. Nanotechnol. 10, 796-803 (2015).

2. Kim, J., Piao, Y. Z. \& Hyeon, T. Multifunctional nanostructured materials for multimodal imaging, and simultaneous imaging and therapy. Chem. Soc. Rev. 38, 372-390 (2009).

3. Carlton, P. M. et al. Fast live simultaneous multiwavelength four-dimensional optical microscopy. Proc. Natl Acad. Sci. USA 107, 16016-16022 (2010).

4. Wrobel, A. T. et al. A fast and selective near-infrared fluorescent sensor for multicolor imaging of biological nitroxyl (HNO). J. Am. Chem. Soc. 136, 4697-4705 (2014)

5. Wang, L. V. Multiscale photoacoustic microscopy and computed tomography. Nat. Photonics 3, 503-509 (2009).

6. Rocha, U. et al. Subtissue thermal sensing based on neodymium-doped LaF3 nanoparticles. ACS Nano 7, 1188-1199 (2013).

7. Miyazaki, J. et al. Simultaneous dual-wavelength imaging of nonfluorescent tissues with 3D subdiffraction photothermal microscopy. Opt. Express 23, 3647-3656 (2015)

8. Miyazaki, J. \& Kobayahsi, T. Photothermal microscopy for high sensitivity and high resolution absorption contrast imaging of biological tissues. Photonics $\mathbf{4}$, 32 (2017)

9. Galanzha, E. I. et al. In vivo magnetic enrichment and multiplex photoacoustic detection of circulating tumour cells. Nat. Nanotechnol. 4, 855-860 (2009).

10. Zharov, V. P. Ultrasharp nonlinear photothermal and photoacoustic resonances and holes beyond the spectral limit. Nat. Photonics 5, 110-116 (2011).
11. Graydon, O. Imaging polarization. Nat. Photonics 7, 343 (2013).

12. Gurjar, R. S. et al. Imaging human epithelial properties with polarized lightscattering spectroscopy. Nat. Med. 7, 1245-1248 (2001).

13. Zhang, C. H. et al. Dual-color single-mode lasing in axially coupled organic nanowire resonators. Sci. Adv. 3, e1700225 (2017).

14. Mukherjee, A., Shim, Y. \& Myong Song, J. Quantum dot as probe for disease diagnosis and monitoring. Biotechnol. J. 11, 31-42 (2016).

15. Huang, L. et al. Composition-graded cesium lead halide perovskite nanowires with tunable dual-color lasing performance. Adv. Mater. 30, 1800596 (2018).

16. $\mathrm{Xu}, J . \mathrm{Y}$. et al. Room-temperature dual-wavelength lasing from singlenanoribbon lateral heterostructures. J. Am. Chem. Soc. 134, 12394-12397 (2012).

17. Liu, Z. C. et al. Dynamical color-controllable lasing with extremely wide tuning range from red to green in a single alloy nanowire using nanoscale manipulation. Nano Lett. 13, 4945-4950 (2013).

18. Yang, Z. Y. et al. Broadly defining lasing wavelengths in single bandgap-graded semiconductor nanowires. Nano Lett. 14, 3153-3159 (2014).

19. Fan, F. et al. Simultaneous two-color lasing in a single CdSSe heterostructure nanosheet. Semiconductor Sci. Technol. 28, 065005 (2013).

20. Zhang, W., Yao, J. N. \& Zhao, Y. S. Organic micro/nanoscale lasers. Acc. Chem. Res. 49, 1691-1700 (2016).

21. Cui, Y. J. et al. Photonic functional metal-organic frameworks. Chem. Soc. Rev. 47, 5740-5785 (2018).

22. Furukawa, $\mathrm{H}$. et al. The chemistry and applications of metal-organic frameworks. Science 341, 1230444 (2013).

23. Kitagawa, S., Kitaura, R. \& Noro, S. Functional porous coordination polymers. Angew. Chem. Int. Ed. 43, 2334-2375 (2004).

24. Feng, L. et al. The chemistry of multi-component and hierarchical framework compounds. Chem. Soc. Rev. 48, 4823-4853 (2019).

25. Wang, $X$. et al. Pore environment engineering in metal-organic frameworks for efficient ethane/ethylene separation. J. Mater. Chem. A 7, 13585-13590 (2019).

26. $\mathrm{He}, \mathrm{H}$. J. et al. Polarized three-photon-pumped laser in a single MOF microcrystal. Nat. Commun. 7, 11087 (2016).

27. Zhang, Y. et al. Dual-wavelength lasing from organic dye encapsulated metal-organic framework microcrystals. Chem. Commun. 55, 3445-3448 (2019)

28. He, H. J. et al. Periodically aligned dye molecules integrated in a single MOF microcrystal exhibit single-mode linearly polarized lasing. Adv. Optical Mater. $\mathbf{5}$, 1601040 (2017).

29. He, H. J. et al. MOF-based organic microlasers. Adv. Optical Mater. 7, 1900077 (2019).

30. $\mathrm{Yu}, \mathrm{J}$. C. et al. Confinement of pyridinium hemicyanine dye within an anionic metal-organic framework for two-photon-pumped lasing. Nat. Commun. 4, 2719 (2013).

31. Li, H. J. et al. Dual-band simultaneous lasing in MOFs single crystals with FabryPerot microcavities. Sci. China Chem. 62, 987-993 (2019).

32. Liu, X. Y. et al. Using a multi-shelled hollow metal-organic framework as a host to switch the guest-to-host and guest-to-guest interactions. Angew. Chem. Int. Ed. 57, 2110-2114 (2018).

33. Jayachandrababu, K. C., Sholl, D. S. \& Nair, S. Structural and mechanistic differences in mixed-linker zeolitic imidazolate framework synthesis by solvent assisted linker exchanc dotge and de Novo routes. J. Am. Chem. Soc. 139, 5906-5915 (2017)

34. Zhou, Z. H. et al. Organic printed core-shell heterostructure arrays: a universal approach to all-color laser display panels. Angew. Chem. Int. Ed. 59, 11814-11818 (2020)

35. Zhu, H. M. et al. Lead halide perovskite nanowire lasers with low lasing thresholds and high quality factors. Nat. Mater. 14, 636-642 (2015). 